\title{
Mixed versus impulse-oriented domain decomposition method for granular dynamics
}

\author{
Damien Iceta — David Dureisseix — Pierre Alart \\ Laboratoire de Mécanique et Génie Civil \\ Université Montpellier 2/CNRS UMR5508, CC048 \\ Place Eugène Bataillon \\ F-34095 Montpellier cedex 5 \\ \{Damien.Iceta, David.Dureisseix,Pierre.Alart\}@lmgc.univ-montp2.fr
}

\begin{abstract}
In this article, we are concerned with the numerical simulation of granular media, characterized as a large scale discrete system, involving non smooth interactions. For such problems, domain decomposition approaches are potential interesting alternative solvers. Herein, two robust and generic monoscale approaches are formulated and compared. The first numerical results reveal a non standard behavior in term of numerical scalability.

RÉSUMÉ. Dans cet article, nous nous intéressons à la simulation numérique des milieux granulaires, caractérisés comme des systèmes discrets de grande taille, avec des interactions non régulières. Pour de tels problèmes, les méthodes de décomposition de domaine sont des candidats intéressants comme solveurs alternatifs. Ici, deux approches monoéchelles robustes et génériques sont formulées et comparées. Les premiers résultats montrent un comportement non standard en termes d'extensibilité numérique.

KEYWORDS: non smooth dynamics, LATIN, NSCD, numerical scalability.

MOTS-CLÉS : dynamique non régulière, LATIN, NSCD, extensibilité numérique.
\end{abstract}

DOI:10.3166/EJCM.18.429-443 @ 2009 Lavoisier, Paris 


\section{Introduction}

The simulation of granular media behavior at small scale (i.e. the size of the grains) leads to the modelling of each individual grain, as well as each interaction between them. Such numerical tests allow to estimate a large number of information (grain movements, chains of forces...) but they also require a huge amount of computational resources. Indeed the number of grains may be large, and the number of interactions is even larger.

Classical approaches embed a rigid model for each grain, and a non smooth unilateral contact, with or without friction, cohesion... as a model of interaction. The resulting problem at small scale is therefore belonging to non smooth dynamics.

A wide range of practical engineering applications (monuments, masonry, blocky rocks, geomaterial, ballasts, powders) concerns quasi-static evolutions of dense granulates and slow flows in which the forces network is the main mechanical feature (Nedderman, 1992; Radjai et al., 1998). The numerical simulations have to be performed using contact dynamic methods based on a fully implicit resolution of the contact forces. At a given step of evolution, all the kinematic constraints within the packing are simultaneously taken into account together with the equations of motions to determine all the contact forces in the packing. This allows to deal properly with nonlocal momentum transfers implied in multiple collisions, contrary to molecular dynamics schemes traditionally used that reduce the system evolution to a succession of binary collisions (Cundall et al., 1979).

To be able to use classical numerical schemes such as molecular dynamics (MD), this non smoothness is often alleviated using penalization techniques (for instance using a contact stiffness). These approaches are usually justified by invocation of physical arguments (grains exhibit a small flexibility, therefore the contact behavior is better described with a Hertzian model, or even with a simplified apparent stiffness). The main counter argument is the following: If the physical contact indeed exhibits a certain stiffness amount, the stiffness is sufficiently high that if used with classical solvers, the physically regularized problem is often stiff enough to impair the convergence and/or the stability of the numerical scheme, and eventually requires drastically reduced time steps. In such cases, the penalizing term has to be artificially reduced to cope with the solver requirements. As a consequence, if one can use a solver suited to non smooth evolutions, the stiff contact (with an infinite rigidity) is therefore a better model than an artificially under-stiffened regularized model.

In this article, we focus on using two non smooth solvers. The first one is derived from the Large Time Increment method (LATIN) initiated by Ladevèze (1999); the second one, developed by Moreau (1999) in the framework of Non Smooth Contact Dynamics (NSCD), may be interpreted as a Non Linear Gauss-Seidel (NLGS) algorithm. For frictionless problems these methods are not the more efficient in comparison with Projected Conjugate Gradient algorithms easily extended from the linear case (Dilintas et al., 1988; May, 1986; Renouf et al., 2004). The Conjugate Gradient schemes are also used by the Domain Decomposition Methods for linear problems 
(BDD (Mandel, 1993), FETI (Farhat et al., 1991)). These methods are difficult to extend to frictional problems - and more yet to more general interactions (adhesion, capillarity) - and the performances are not so good; indeed the conjugate gradient approach in this case exhibits convergence difficulties (Renouf et al., 2004). This is the reason why we develop here a Domain Decomposition strategy using only robust and generic incremental solvers based on LATIN and NLGS.

The 'direct' extension of FETI-like methods (using an active set approach) for frictionless (Dureisseix et al., 2001; Avery et al., 2004; Avery et al., 2009) or frictional contact problems (Rebel et al., 2003), as well as the approaches of Dostál for frictionless (Dostál et al., 1998; Dostál et al., 2000), or frictional contact problems (Haslinger et al., 2002; Dostál et al., 2005), exhibited their efficiencies for 'assembling'-like problems (with a few number of contact interfaces), but have the same limitations as the previous ones for diffuse contact conditions (with a large number of frictional contact conditions).

\section{Non smooth dynamics model for granular media without friction}

We consider interactions between grains, that constitute a system which can be viewed as a rigid multibody collection of particles. For sake of simplicity, unilateral contact without friction is considered herein.

\subsection{The grain dynamics}

We assume that each grain denoted with $i$ interacts with its neighbors via a finite collection of punctual contacts. Its mass is denoted with $m_{i}$, and its center of mass with $G_{i}$. Its inertial operator at point $G_{i}$ is $J_{i G}$. At the contact labelled $\alpha$ and located at point $M_{\alpha}$, the two contacting bodies are conventionally called the candidate contactor and the antagonist one. At the point $M_{\alpha}$ the common normal unit vector $n_{\alpha}$ is directed toward the candidate body.

The body kinematics is described with 6 parameters in 3D: 3 are the elements of the celerity vector of the mass center $U_{i}$, the others are the elements of the rotation rate $\Omega_{i}$.

In order to compute the evolution in time, an integration scheme is needed. Since the behavior of a granular medium involves non smooth effects in velocity jumps, a scheme which is not built on the derivability and continuity of velocity equations is used. Because of the very high number of contacts involved in dense granular media, a Time-Stepping scheme is chosen rather than an Event-Driven one, which would lead to a too small time step. In particular, the Event-Driven scheme is unable to take into account simultaneous contacts (Glocker et al., 1995; Liu et al., 2008; Liu et al., 2009). 
A Time-Stepping scheme provides a discretized equation of dynamics involving impulses in its right-hand side, i.e. the integral over $] t^{-}, t^{+}[$of the force and moment quantities. The kinematic quantities have to be determined at the end of the step $t^{+}$,

$$
\left\{\begin{array}{c}
m_{i}\left(U_{i}^{+}-U_{i}^{-}\right) \\
J_{i G}\left(\Omega_{i}^{+}-\Omega_{i}^{-}\right)
\end{array}\right\}_{G}=\left\{\begin{array}{c}
P_{i}+P_{i}^{d} \\
K_{i}+K_{i}^{d}
\end{array}\right\}_{G}
$$

where the external actions applied on the system are: the contact impulse resultant $P_{i}$, the non contact impulse resultant $P_{i}^{d}=\int_{t^{-}}^{t^{+}} F_{i}^{d} d t$ (where $F_{i}^{d}$ is the non contact resultant), the contact impulse moment at $G_{i}, K_{i}$, the non contact impulse moment at $G_{i}, K_{i}^{d}=\int_{t^{-}}^{t^{+}} M_{i}^{d} d t$ (where $M_{i}^{d}$ is the non contact moment at $G_{i}$ ).

Assembling mass and inertia in a small local matrix $M_{i}$, the kinematic parameters $U_{i}$ and $\Omega_{i}$ in a small local vector $V_{i}$, the impulse quantities $P_{i}$ and $K_{i}$ in a vector $R_{i}$, and finally the given quantities $P_{i}^{d}$ and $K_{i}^{d}$ into a vector $R_{i}^{d}$, the dynamical admissibility reads:

$$
M_{i} V_{i}^{+}=R_{i}+R_{i}^{d}+M_{i} V_{i}^{-}
$$

For simplicity the upperscript + is omitted in the following. These vectors, associated to each grain, may be concatenated in global vectors, in such a way the dynamics of the granular medium is rewritten as the following vector equation where the matrix $M$ is diagonal, provided that the global coordinates of rotation vectors are expressed in the inertia eigen basis of each grain.

$$
M V=R+R^{d}+M V^{-}
$$

\subsection{Grain to contact mapping and reverse}

The relative velocity of the candidate body with regards to the antagonist one equals the vector difference of the normal velocities of the respective contacting particles, say $v^{\alpha}=H^{\alpha t} V$, where $H^{\alpha t}$ denotes a linear mapping from the particle level to the contact one. In a view of the definition of generalized components, the normal contact force $r^{\alpha}$ exerted by antagonist body upon the candidate one contributes in the element $R: R=H^{\alpha} r^{\alpha}$.

The relative velocities of all contacts and the contact impulses are concatenated in $v=H^{t} V$ and $R=H r$.

\subsection{Constitutive relation of an interaction}

We focus in this article on simple unilateral contact which is naturally expressed as a complementary condition linking contact force to gap. For dynamics, Moreau (1999) proved via a viability lemme that we can use a velocity-impulse complementary law. 
The unilaterality of interaction is written :

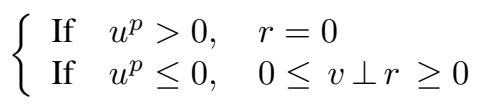

where $u^{p}$ is the gap prediction (positive if no contact is detected); we use here the classical 'leapfrog' predictor (Moreau, 1999): $u^{p}=u^{-}+\frac{h}{2} v^{-}$, with $h=t^{+}-t^{-}$as the time step. Such a relation is summarized in the following formal equation,

$$
\mathcal{R}(v, r)=0
$$

\subsection{Reference problem}

Taking into account the grain dynamics [3] and the contact to grain mapping, the impulse-oriented formulation of the reference problem can be obtained (called reduced dynamics equation):

$$
v-W r=v^{d}+v^{-}
$$

where $W$ is the Delassus operator $W=H^{t} M^{-1} H$, and the given quantities are $v^{d}=H^{t} M^{-1} R^{d}$ and $v^{-}=H^{t} V^{-}$.

When velocities are prescribed on a part $\Omega_{1}$ of the grains: $\left.V\right|_{\Omega_{1}}=V_{d}$, the grain degrees of freedom can be splitted into two sets: with the subscript $u$ the unknown values, and with the subscript $p$ the prescribed values. The reduced dynamics equation [6] then reads:

$$
v-W_{u} r=\tilde{v}^{d}+\tilde{v}^{-}
$$

with $W_{u}=H_{u}^{t} M_{u}^{-1} H_{u}, \tilde{v}^{d}=H_{u}^{t} M_{u}^{-1} R_{u}^{d}+H_{p}^{t} V_{p}$, and $\tilde{v}^{-}=H_{u}^{t} V_{u}^{-}$. Since this problem has the same structure as [6], and to simplify the notations, [6] will be used in the following. To close the problem, one has to add the constitutive relation [5].

\section{Domain splitting}

The suited splitting of a structure for using a domain decomposition approach is a geometrical partitioning of the domain occupied by this structure. In the case of a discrete structure, there are two common ways to partition it: (i) distribute the elements, the links, or the interactions among substructures, or (ii) distribute the nodes or the grains among substructures. Both strategies were used in the literature. For a topological point of view, they correspond to the splitting of two different graphs: the connectivity graph or its dual graph. The node or grain distribution if often considered in algebraic partitioning where the left hand side matrix of the velocity formulation 
is splitted according to its columns (degrees of freedom). In our case, since the non smoothness may occur in the interactions between grains, we choose to distribute these interactions among the substructures (we proceed by distributing the middle points between the centers of mass of interacting grains, according to their coordinates, with the help of an arbitrary regular underlying grid, Figure 11. Indeed, with such a choice, some of the 'boundary' grains are splitted themselves in the data structures arising for the subdomains. Note that for dynamical evolutions, contrary to (Alart et al., 2008), the mass located in such a grain has to be splitted in two contributions, one for each duplicated grain, see Figure 1. The interface between two subdomains is defined to be the set of these grains, joining a substructure to the other. The nonsmoothness is therefore localized within the substructures only. This modeling choice is identical to (Barboteu et al., 2001) and somehow the dual of the one proposed in (Ladevèze $e t$ al., 2002) where the non-linearities (contact of crack lips) are isolated in the interfaces.

Introducing such a splitting leads to have additional unknown fields to emerge: on each interface between a subdomain $\Omega_{E}$ and a subdomain $\Omega_{E^{\prime}}$, a discrete repartition of impulsion $F_{E E^{\prime}}$ expressing the actions of the subdomain $\Omega_{E^{\prime}}$ onto the subdomain $\Omega_{E}$. A discrete velocity field $V_{E E^{\prime}}$ is the trace of the velocity field in the subdomain $\Omega_{E}$ on its local interface with the neighboring subdomain $\Omega_{E^{\prime}}$. With the previous choice of splitting, the behavior of the interface is perfect, i.e. it transfers both impulse (the splitted grains interforce) and velocity (of centers of mass):

$$
\begin{aligned}
& F_{E E^{\prime}}+F_{E^{\prime} E}=0 \\
& V_{E E^{\prime}}=V_{E^{\prime} E}
\end{aligned}
$$

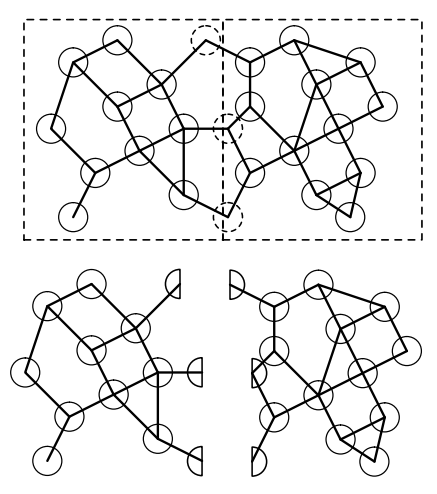

Figure 1. Geometrical partitioning of the domain

The data structure of the dynamical reference problem is therefore complexified with the additional interface quantities. In particular, for a subdomain $E$ among $n_{S D}$ subdomains, the subscript $E \Gamma$ will denote the collection of its local interfaces (i.e. its neighboring interfaces). $F_{E \Gamma}$ will therefore be a vector that collects the impulse 
on these local interfaces, and $V_{E \Gamma}$ will collect the velocity on the same local interfaces. Moreover, one needs a Boolean matrix that maps the local interfaces degrees of freedom to the subdomain degrees of freedom; this will be denoted with $C_{E \Gamma}$ in the following.

\section{Domain decomposition solvers}

Two numerical strategies, using the same database structure issued from the decomposition of Section 3, are detailed and compared in the following.

\subsection{Monoscale LATIN method}

In the present case of an incremental approach, and for non evolutionary problems, the Large Time Increment method (Ladevèze, 1999) is close to an Augmented Lagrangian approach (Alart et al., 2005). It is an iterative method, which at each iteration requires two different steps, namely the local stage, dealing with local constitutive relations, and the linear stage, dealing with admissibility conditions. This iterative scheme successively builds approximations of the solution, i.e. the normal relative velocity and the impulse $\left(v_{E}, r_{E}\right), E=1, \ldots, n_{S D}$, and the nodal velocity and impulse on each interface $\left(V_{E E^{\prime}}, F_{E E^{\prime}}\right)$.

\subsubsection{Local stage}

Once $\left(v_{E}, r_{E}\right)$ and $\left(V_{E E^{\prime}}, F_{E E^{\prime}}\right)$ are known, the local stage consists in finding $\left(\widehat{v}_{E}, \widehat{r}_{E}\right)$ and $\left(\widehat{V}_{E E^{\prime}}, \widehat{F}_{E E^{\prime}}\right)$ satisfying:

- for each interface, the interface behavior:

$$
\left\{\begin{array}{l}
\widehat{F}_{E E^{\prime}}+\widehat{F}_{E^{\prime} E}=0 \\
\widehat{V}_{E E^{\prime}}=\widehat{V}_{E^{\prime} E}
\end{array}\right.
$$

- the interface search direction:

$$
\left(\widehat{F}_{E E^{\prime}}-F_{E E^{\prime}}\right)-d^{+}\left(\widehat{V}_{E E^{\prime}}-V_{E E^{\prime}}\right)=0
$$

- for each interaction of each subdomain, the constitutive relation $\mathcal{R}\left(\widehat{v}_{E}, \widehat{r}_{E}\right)=0$;

- and the interaction search direction:

$$
\left(\widehat{r}_{E}-r_{E}\right)-l^{+}\left(\widehat{v}_{E}-v_{E}\right)=0
$$

$d^{+}$and $l^{+}$are two positive parameters of the method. For perfect interfaces and frictionless contact interactions, the solution of the local stage is explicit:

$$
\left\{\begin{array}{l}
\widehat{F}_{E^{\prime} E}=-\widehat{F}_{E E^{\prime}}=\frac{1}{2}\left[\left(F_{E^{\prime} E}-F_{E E^{\prime}}\right)-d^{+}\left(V_{E^{\prime} E}-V_{E E^{\prime}}\right)\right] \\
\widehat{V}_{E E^{\prime}}=\widehat{V}_{E^{\prime} E}=\frac{1}{2}\left[\left(V_{E^{\prime} E}+V_{E E^{\prime}}\right)-d^{+}\left(F_{E^{\prime} E}+F_{E E^{\prime}}\right)\right]
\end{array}\right.
$$




$$
\begin{cases}\text { If } u^{p}>0, & \widehat{r}_{E}=0 \text { and } \widehat{v}_{E}=v_{E}-\left(l^{+}\right)^{-1} r_{E} \\ \text { If } u^{p} \leq 0, & \widehat{r}_{E}=<r_{E}-l^{+} v_{E}>_{+} \text {and } \\ & \widehat{v}_{E}=-\left(l^{+}\right)^{-1}<r_{E}-l^{+} v_{E}>_{-}\end{cases}
$$

\subsubsection{Linear stage}

Once $\left(\widehat{v}_{E}, \widehat{r}_{E}\right)$ and $\left(\widehat{V}_{E E^{\prime}}, \widehat{F}_{E E^{\prime}}\right)$ are known, the linear stage consists in finding $\left(v_{E}, r_{E}\right)$ and $\left(V_{E E^{\prime}}, F_{E E^{\prime}}\right)$ satisfying:

- for each subdomain, its dynamical evolution:

$$
M_{E} V_{E}=R_{E}+F_{E}+R_{E}^{d}+M_{E} V_{E}^{-}
$$

and the remaining admissibility equations: $v_{E}=H_{E}^{t} V_{E}, R_{E}=H_{E} r_{E}, F_{E}=$ $C_{E \Gamma}^{t} F_{E \Gamma}, V_{E \Gamma}=C_{E \Gamma} V_{E}$.

- for each subdomain, and its local interfaces, the search directions:

$$
\left\{\begin{array}{l}
\left(r_{E}-\widehat{r}_{E}\right)+l^{-}\left(v_{E}-\widehat{v}_{E}\right)=0 \\
\left(F_{E \Gamma}-\widehat{F}_{E \Gamma}\right)+d^{-}\left(V_{E \Gamma}-\widehat{V}_{E \Gamma}\right)=0
\end{array}\right.
$$

$d^{-}$and $l^{-}$are also two positive parameters of the method. Using the search directions, the linear global problem reads on each subdomain:

$$
\left(M_{E}+H_{E} l^{-} H_{E}^{t}+C_{E \Gamma}^{t} d^{-} C_{E \Gamma}\right) V_{E}=\widehat{R}_{E}+R_{E}^{d}+M_{E} V_{E}^{-}
$$

where $\widehat{R}_{E}=H_{E}\left(\widehat{r}_{E}+l^{-} \widehat{v}_{E}\right)+C_{E \Gamma}^{t}\left(\widehat{F}_{E \Gamma}+d^{-} \widehat{V}_{E \Gamma}\right)$ is a given quantity at this stage. Once $V_{E}$ is obtained, the other quantities are obtained in a post-processing step:

$$
\begin{aligned}
& v_{E}=H_{E}^{t} V_{E} \\
& r_{E}=\widehat{r}_{E}+l^{-} \widehat{v}_{E}-l^{-} H_{E}^{t} V_{E} \\
& F_{E \Gamma}=\widehat{F}_{E \Gamma}+d^{-} \widehat{V}_{E \Gamma}-d^{-} V_{E \Gamma}
\end{aligned}
$$

\subsection{Monoscale NLGS method}

The continuity condition of the velocity field on the global interface $\Gamma$ requires Boolean signed operators $A_{E \Gamma}$ that extract from a subdomain vector $V_{E}$ its signed restriction to the interface,

$$
\sum_{E} A_{E \Gamma} V_{E}=0
$$

We define $F_{\Gamma}$ as the multiplier associated to this constraint. The dynamics of each subdomain according to [6] is described by

$$
W_{E} r_{E}-v_{E}-H_{E}^{t} M_{E}^{-1} A_{E \Gamma}^{t} F_{\Gamma}=-v_{E}^{d}-v_{E}^{-}
$$


The combination of the dynamics [22], the continuity condition [21] and the constitutive relations of the interactions restricted to subdomains [5] gives,

$$
\left\{\begin{array}{c}
W_{E} r_{E}-v_{E}-H_{E}^{t} M_{E}^{-1} A_{E \Gamma}^{t} F_{\Gamma}=-v_{E}^{d}-v_{E}^{-} \\
\mathcal{R}\left(v_{E}, r_{E}\right)=0 \\
\sum_{E} A_{E \Gamma} V_{E}=0
\end{array}\right\} E=1, \ldots, n_{S D}
$$

Using directly the dynamics equation [3] per subdomain the last equation may be replaced by an equation with $F_{\Gamma}$ as the main unknown $\left(X=\sum_{E} A_{E \Gamma} M_{E}^{-1} A_{E \Gamma}^{t}\right)$,

$$
\left\{\begin{array}{c}
W_{E} r_{E}-v_{E}-H_{E}^{t} M_{E}^{-1} A_{E \Gamma}^{t} F_{\Gamma}=-v_{E}^{d}-v_{E}^{-} \\
\mathcal{R}\left(v_{E}, r_{E}\right)=0 \\
X F_{\Gamma}-\sum_{E} A_{E \Gamma} M_{E}^{-1} R_{E}=\sum_{E} A_{E \Gamma} M_{E}^{-1} R_{E}^{d}
\end{array}\right\} E=1, \ldots, n_{S D}
$$

A Gauss-Seidel like algorithm applied to this last system consists of two steps. The first one performs one Gauss-Seidel iteration for each subdomain indiced by $E$,

$$
\left\{\begin{array}{l}
W_{E}^{L} r_{E}-v_{E}=H_{E}^{t} M_{E}^{-1} A_{E \Gamma}^{t} \tilde{F}_{\Gamma}-v_{E}^{d}-\left(W_{E}-W_{E}^{L}\right) \tilde{r}_{E}-v_{E}^{-} \\
\mathcal{R}\left(v_{E}, r_{E}\right)=0
\end{array}\right.
$$

The classical Gauss-Seidel splitting of $W_{E}$ uses the lower triangular part $W_{E}^{L}$ (including the diagonal part $\left.W_{E}^{D}\right): W_{E}=W_{E}^{L}+\left(W_{E}-W_{E}^{L}\right)$. This first step is then performed subdomain per subdomain and may be easily parallelized. The non linear problem 25 is easily solved, progressing whith each unknown couple $\left(v_{E}^{\alpha}, r_{E}^{\alpha}\right.$, one after the other, for which a graph intersection technique provide the result explicitely (for the frictionless case).

After updating the impulse quantities grain per grain $R_{E}$ the second step consists in solving the last equation of the system [24],

$$
X F_{\Gamma}=\sum_{E} A_{E \Gamma} M_{E}^{-1}\left(R_{E}+R_{E}^{d}\right)
$$

This second step benefits from the diagonal feature of the $X$ matrix, that is a specific issue of the dynamics of rigid grains.

\subsection{Algorithmic connection of the two methods}

To compare the two previous methods, one has to specialize each of them (with respect to the free choices that have been made during their design). Moreover, concern- 
ing notations, the Boolean mapping matrices are linked with $C_{E \Gamma}=\left|A_{E \Gamma}\right|$ and the subdomain impulse arising from the global interface are $F_{E}=C_{E \Gamma}^{t} F_{E \Gamma}=A_{E \Gamma}^{t} F_{\Gamma}$ whenever the impulse equilibrium of interfaces is enforced.

First, one has to consider a Jacobi version of the previous Gauss-Seidel approach, i.e. choosing the diagonal part $W_{E}^{D}$ in the splitting: $W_{E}=W_{E}^{D}+\left(W_{E}-W_{E}^{D}\right)$. In such a way, shifting from Gauss-Seidel to Jacobi, the convergence of NLGS is expected to decrease.

Second, a degenerated choice of search direction parameters of the LATIN method has to be selected in order to identify the two resulting algorithms: the search directions of the local stage should be $d^{+}=\frac{1}{2} X^{-1}$ and $l^{+}=\left(W_{E}^{D}\right)^{-1}$, and the search directions of the linear stage should be 'vertical', i.e. $d^{-}=l^{-}=0$. With these choices, the convergence of the LATIN method is expected to decrease also.

The unification of the two previous algorithms is therefore subjected to a degeneracy of both of them.

\section{Basic behaviors in linear case}

To test the scalability of the methods we deal here with a linear model example without realistic relevance, but considered as an intermediate problem before tackling granular systems. A granular sample is performed using the numerical plateform LMGC9 1 dedicated to multicontact problems (Jean, 1999). The contact interactions, originally written with a velocity-impulse formulation, are replaced by linear viscouslike links between the centers of the grains. In this way, the example may be viewed as a truss composed of massive nodes and damped links. The only adaptation to the reference problem is the constitutive relation $\mathcal{R}(v, r)=0$ that is now: $r=-k v(k$ is the viscosity term multiplied by the time step). The tested sample is composed of 5191 grains, Figure 2, the velocity of bottom grains is prescribed to zero, while left and right sides are subjected to a global rotation to mimic a global shearing. In such a case, the reference problem can be further simplified into a single equation, that reads: $\left(W+k^{-1}\right) r=-v_{d}-v^{-}$. The left hand side is therefore composed of a diagonal, regular, viscous-like part, and the Delassus operator, which is singular (in most cases: indeed, imagine replacing the contact interactions with bars of a truss, the size of the kernel of the Delassus operator is the number of self-stress states). To check the influence of this singular character, two different values of $k$ will be tested: a reference value $k_{0}$ selected as the mean value of $\left(W^{D}\right)^{-1}$, and a value $k_{1}$ reducing the regularizing contribution of viscosity: $k_{1}=50 k_{0}$.

Several convergence curves are plotted in each case, for a single time step increment of the problem, see Figures 3 and 4 for each method, and for different numbers of subdomains, i.e. $n_{S D}=1,2,4,8,16$ or 32 . For this linear test problem, and con-

1. http://www.lmgc.univ-montp2.fr/ dubois/LMGC90/ 


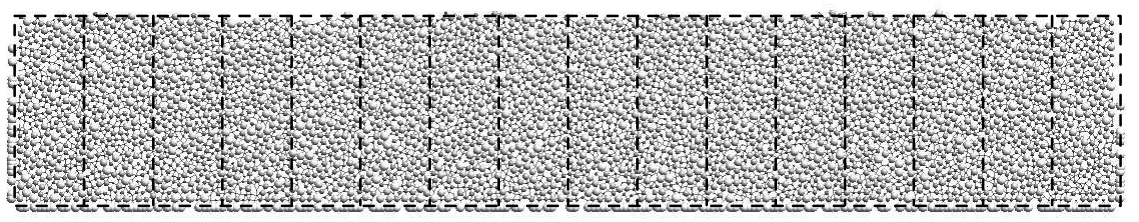

Figure 2. Test case with 5191 nodes and 10177 interactions, and the grid used to split the domain into 16 subdomains

trary to the granular one, the solution is unique in terms of impulses $r_{\text {ref }}$. Consequently, a relative error is used to check the convergence:

$$
\eta=\sqrt{\frac{\left(r-r_{\text {ref }}\right)^{t}\left(r-r_{\text {ref }}\right)}{\left(r+r_{\text {ref }}\right)^{t}\left(r+r_{\text {ref }}\right)}}
$$

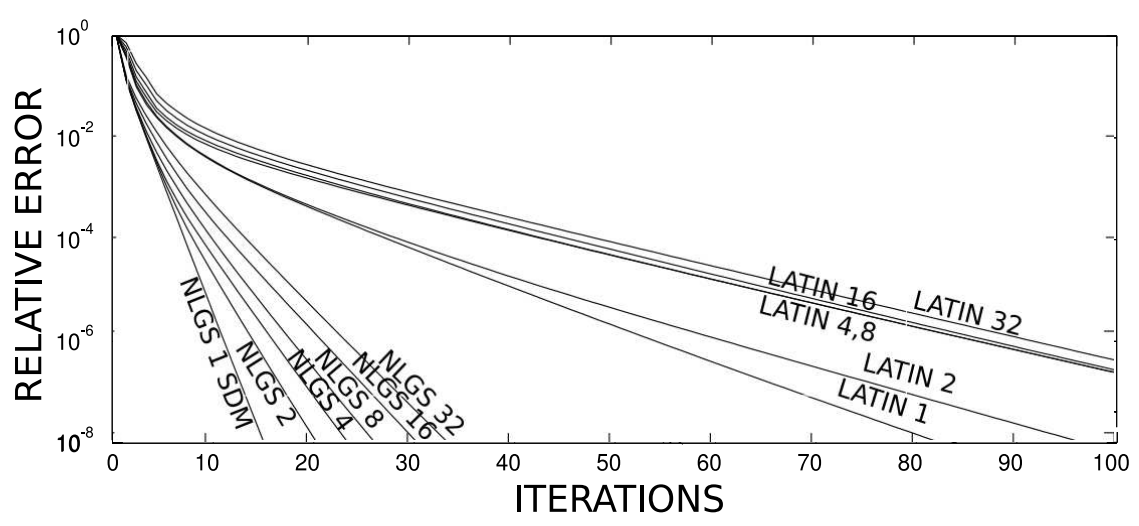

Figure 3. Results with $k=k_{0}$

For the LATIN method, the search direction parameters are chosen as $l^{+}=l^{-}=k$ and $d^{+}=d^{-}=k$.

As a general trend, the convergence rates of both methods decrease when the influence of the regular viscosity part decreases. On this test, NLGS exhibits a higher convergence rate than the LATIN, though it is less obvious for the less regular case (Figure 4).

The numerical scalability is lost (when the number of subdomain increases) but it is not systematic (contrary to the known results for a continuum media case). Indeed, for the LATIN method, for $k=k_{0}$, the 4, 8, 16 and 32 subdomain cases are very similar; for $k=k_{1}$, the 1,2, 4 and 8 subdomain cases on one hand, and the 16 and 32 subdomain cases on the other hand are similar. 
440 European Journal of Computational Mechanics. Volume 18 - No. 5-6/2009

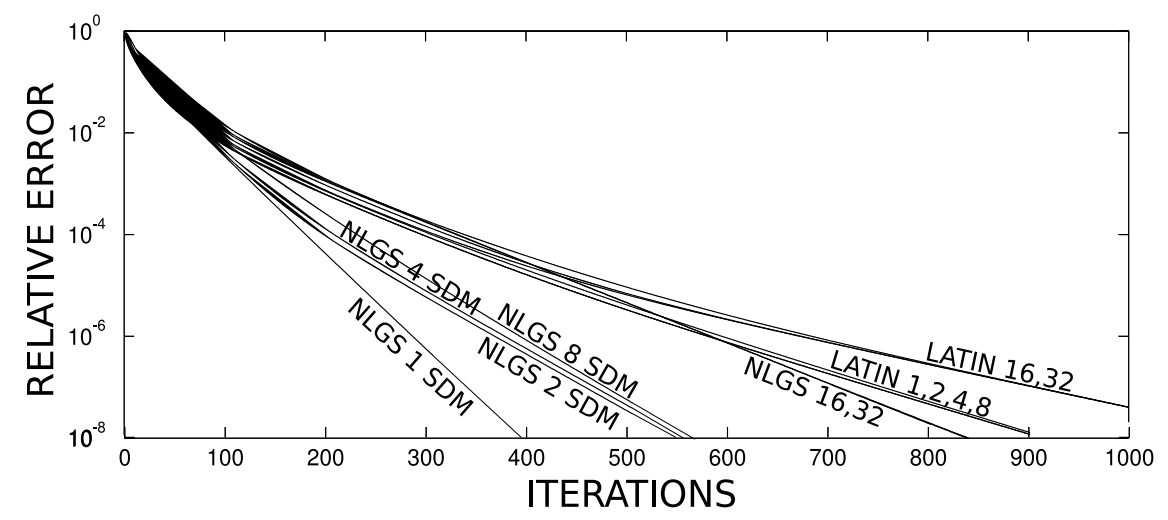

Figure 4. Results with $k=k_{1}=50 k_{0}$

\section{First results for granular media}

A smaller problem with 991 grains and 1679 non smooth interactions (Figure 5) issued from LMGC90 platform is this time considered with frictionless contact interactions [4]. Since the solution is not unique in this case, the relative error is replaced with a relative error indicator to check the convergence: at iteration $i$,

$$
\zeta_{i}=\sqrt{\frac{\left(r_{i}-r_{i-1}\right)^{t}\left(r_{i}-r_{i-1}\right)}{r_{i}^{t} r_{i}}}
$$

Figure 6 plots its evolution along iterations, for a single time step increment of the problem, for both methods and 1 to 8 subdomains. At least for this test case, all the convergences are similar.

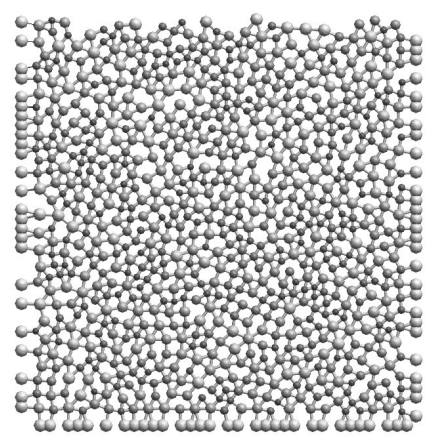

Figure 5. Test case with 991 nodes and 1679 interactions 


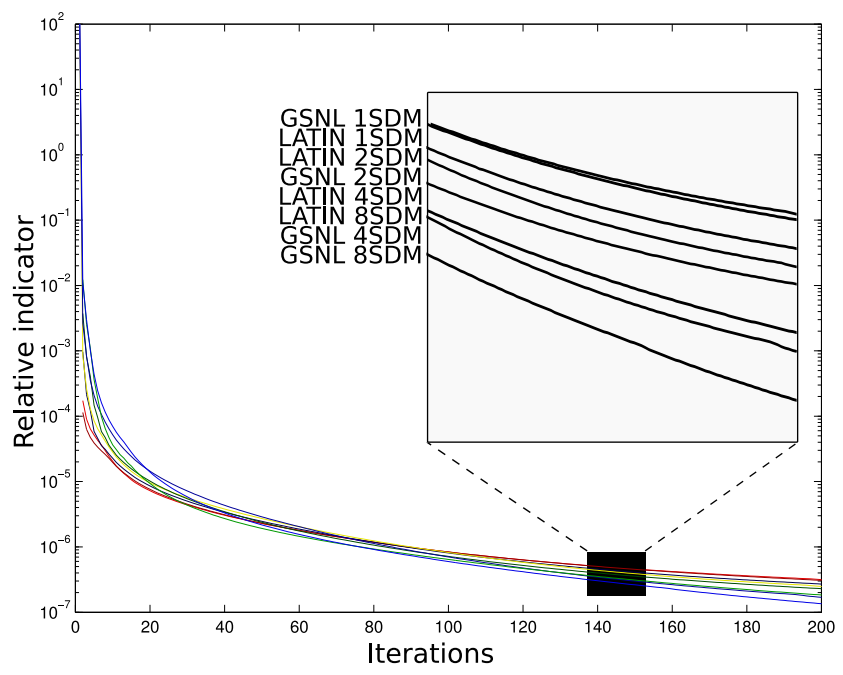

Figure 6. Results for non linear case

\section{Conclusion}

The proposed methods developed in this article seem to be more and more scalable when the interaction behavior exhibits more and more non linearities/non smoothness, even without using a multilevel enrichment. This could be attributed for one part to the simplicity of the non linear solvers (based on a fixed point technique) when compared to conjugate gradient algorithms (Renouf et al., 2004; Dureisseix et al., 2001). For the other part, this may arise from the simultaneous treatment of the non linearities and the domain decomposition, since the most efficient methods embed a domain decomposition for the linear resolutions in outer Newton iterations (De Roeck et al., 1992; Barboteu et al., 2001). Notice that this last approach is not suited to the problem we wish to tackle here, where the non smoothness is diffuse (everywhere in the domain) (Nineb et al., 2007).

Using multiscale versions of the present approaches would nevertheless present several advantages. The first one is to get access to a numerically homogenized behavior of the subdomains, a useful feature to be able to couple two models at two different scales (Mobasher Amini et al., 2009). The second one is the possibility to initialize successive steps in a problem of evolution with a coarse solution provided by the previous time step, in order to accelerate the resolution. The main outlook of this work is nevertheless to tackle the frictional contact case. 
442 European Journal of Computational Mechanics. Volume 18 - No. 5-6/2009

\section{References}

Alart P., Dureisseix D., "A scalable multiscale LATIN method adapted to nonsmooth discrete media", Computer Methods in Applied Mechanics and Engineering, vol. 197, n 5, p. 319331, 2008.

Alart P., Dureisseix D., Renouf M., "Using nonsmooth analysis for numerical simulation of contact mechanics", Nonsmooth Mechanics and Analysis: Theoretical and Numerical Advances, vol. 12 of Advances in Mechanics and Mathematics, Kluwer Academic Press, chapter 17, p. 195-207, 2005.

Avery P., Farhat C., "The FETI family of domain decomposition methods for inequalityconstrained quadratic programming: Application to contact problems with conforming and nonconforming interfaces", Computer Methods in Applied Mechanics and Engineering, 2009. In Press, Accepted Manuscript.

Avery P., Rebel G., Lesoinne M., Farhat C., “A numerically scalable dual-primal substructuring method for the solution of contact problems - Part I: The frictionless case", Computer Methods in Applied Mechanics and Engineering, vol. 193, p. 2403-2426, 2004.

Barboteu M., Alart P., Vidrascu M., "A domain decomposition strategy for nonclassical frictional multi-contact problems", Computer Methods in Applied Mechanics and Engineering, vol. 190, p. 4785-4803, 2001.

Cundall P. A., Stack O. D. L., "A discrete numerical model for granular assemblies”, Geotechnique, vol. 29, $\mathrm{n}^{\circ} 1$, p. 47-65, 1979.

De Roeck Y.-H., Le Tallec P., Vidrascu M., “A domain decomposed solver for nonlinear elasticity", Computer Methods in Applied Mechanics and Engineering, vol. 99, p. 187-207, 1992.

Dilintas G., Laurent-Gengoux P., Trystram D., "A conjugate projected gradient method with preconditioning for unilateral contact problems", Computers and Structures, vol. 29, n 4 , p. $675-680,1988$.

Dostál Z., Friedlander A., Santos S., "Solution of coercive and semicoercive contact problems by FETI domain decomposition”, Contemporary Mathematics, vol. 218, p. 82-93, 1998.

Dostál Z., Gomes Neto F. A. M., Santos S. A., "Solution of contact problems by FETI domain decomposition with natural coarse space projection", Computer Methods in Applied Mechanics and Engineering, vol. 190, n 13-14, p. 1611-1627, 2000.

Dostál Z., Horák D., Kucera R., Vondrák V., Haslinger J., Dobiás J., Pták S., "FETI based algorithms for contact problems: scalability, large displacements and 3D Coulomb friction", Computer Methods in Applied Mechanics and Engineering, vol. 194, $\mathrm{n}^{\circ}$ 2-5, p. 395-409, 2005.

Dureisseix D., Farhat C., "A numerically scalable domain decomposition method for the solution of frictionless contact problems", International Journal for Numerical Methods in Engineering, vol. 50, $\mathrm{n}^{\circ}$ 12, p. 2643-2666, 2001.

Farhat C., Roux F.-X., "A method of finite element tearing and interconnecting and its parallel solution algorithm", International Journal for Numerical Methods in Engineering, vol. 32, $\mathrm{n}^{\circ}$ 6, p. 1205-1227, 1991.

Glocker C., Pfeiffer F., "Multiple impacts with friction in rigid multibody systems.", Nonlinear Dynamics, Transactions of A.S.M.E., vol. 7, n 4, p. 471-497, 1995. 
Haslinger J., Dostál Z., Kucera R., "On a splitting type algorithm for the numerical realization of contact problems with Coulomb friction", Computer Methods in Applied Mechanics and Engineering, vol. 191, n 21-22, p. 2261-2281, 2002.

Jean M., "The non-smooth contact dynamics method", Computer Methods in Applied Mechanics and Engineering, vol. 177, p. 235-257, 1999.

Ladevèze P., Nonlinear computational structural mechanics - New approaches and nonincremental methods of calculation, Springer Verlag, 1999.

Ladevèze P., Nouy A., Loiseau O., "A multiscale computational approach for contact problems", Computer Methods in Applied Mechanics and Engineering, vol. 191, p. 4869-4891, 2002.

Liu C., Zhao Z., Brogliato B., "Frictionless multiple impacts in multibody systems. I. Theoretical framework", Proceedings of the Royal Society A: Mathematical, Physical and Engineering Science, vol. 464, n 2100, p. 3193-3211, 2008.

Liu C., Zhao Z., Brogliato B., "Frictionless multiple impacts in multibody systems. II. Numerical algorithm and simulation results", Proceedings of the Royal Society A: Mathematical, Physical and Engineering Science, vol. 465, n² 2101, p. 1-23, 2009.

Mandel J., "Balancing domain decomposition", Communications in Applied Numerical Methods, vol. 9, p. 233-241, 1993.

May H.-O., "The conjugate gradient method for unilateral problems", Computers and Structures, vol. 12, n 4, p. 595-598, 1986.

Mobasher Amini A., Dureisseix D., Cartraud P., "Multi-scale domain decomposition method for large scale structural analysis with a zooming technique: Application to plate assembly", International Journal for Numerical Methods in Engineering, vol. 79, n 4, p. 417-443, 2009.

Moreau J. J., "Numerical aspects of sweeping process", Computer Methods in Applied Mechanics and Engineering, vol. 177, p. 329-349, 1999.

Nedderman R., Statics and kinematics of granular materials, Cambridge university Press, Cambridge, 1992.

Nineb S., Alart P., Dureisseix D., "Domain decomposition approach for nonsmooth discrete problems, example of a tensegrity structure", Computers and Structures, vol. 85, $\mathrm{n}^{\circ} 9$, p. 499-511, 2007.

Radjai F., Wolf D. E., Jean M., Moreau J. J., "Bimodal character of stress transmission in granular packings", Physical Review Letters, vol. 80, n 1, p. 61-64, 1998.

Rebel G., Farhat C., Lesoinne M., Avery P., “A scalable Dual-Primal domain decomposition method for the solution of contact problems with friction", 7th U.S. National Congress on Computational Mechanics - USNCCM7, 2003.

Renouf M., Alart P., "Conjugate gradient type algorithms for frictional multi-contact problems: Applications to granular materials", Computer Methods in Applied Mechanics and Engineering, vol. 194, p. 2019-2041, 2004.

Received: 9 February 2009

Accepted: 20 May 2009 
